\title{
In Vitro Inhibition of Commercial Douche Products Against Vaginal Microflora
}

\author{
S.I. Pavlova and L. Tao* \\ Department of Oral Biology, College of Dentistry, University of Illinois at Chicago, Chicago, IL
}

\begin{abstract}
Recently, vaginal douching has been associated with many health risks in women. The aim of this study was to analyze the effect of commercial douche products against various vaginal microorganisms, including lactobacilli. Seven commercial douches were tested against eight Lactobacillus clinical isolates and three type strains from the American Type Culture Collection. BV-associated bacteria included six strains of five genera: Gardnerella, Mobiluncus, Mycoplasma, Peptostreptococcus, and Ureaplasma. Two isolates of group B Streptococcus, and three species of Candida were also tested. The minimal inhibition concentrations and minimal contact times for these products against vaginal microorganisms were determined in broth cultures. Four antiseptic-containing douche products showed a strong inhibitory effect against all vaginal microorganisms tested with a short contact time (less than $1 \mathrm{~min}$ ). Three vinegar-containing douche products selectively inhibited vaginal pathogens associated with bacterial vaginosis, group $B$ streptococcal vaginitis, and candidiasis, but not lactobacilli. The antimicrobial effects of the commercial douche products varied among different brands and microbial species tested. Infect. Dis. Obstet. Gynecol. 8:99-104, $2000 . \bigcirc 2000$ Wiley-Liss, Inc.
\end{abstract}

KEY WORDS

bacterial vaginosis; lactobacilli; Candida, group B streptococci

$T$ he vaginal ecological system constitutes a large population of diverse microorganisms. ${ }^{1}$ Under healthy conditions, lactobacilli dominate this population, preventing other potentially harmful microorganisms from colonization or overgrowth. ${ }^{2}$ Lactobacilli produce factors including lactic acid, hydrogen peroxide, and various bacteriocins, which may tend to exclude other microbes in the same environment. Therefore, factors that inhibit or eliminate lactobacilli may facilitate colonization or overgrowth of potentially pathogenic microorganisms in the vagina.

Vaginal douching has a long history and is practiced regularly by many American women and ado- lescent girls. ${ }^{3-6}$ However, little is known about the risks versus personal benefits of this practice. Many clinical studies suggest a possible association between douching and various health risks in women. These include pelvic inflammatory disease, ${ }^{7,8}$ bacterial vaginosis (BV), ${ }^{9-11}$ chlamydia, ${ }^{12}$ and other sexually transmitted infections including HIV, ${ }^{13-15}$ miscarriages ${ }^{16}$ premature birth of low birth-weight infants, ${ }^{17}$ ectopic pregnancy, ${ }^{18,19}$ and cervical cancer. $^{20}$

Although most published data argue against douching, ${ }^{7-20}$ two studies indicated that douching might be medically beneficial. ${ }^{21-22}$ First, douching may significantly eliminate semen after sexual in-

Grant sponsor: Concerned Parents for AIDS Research-AmFAR; Grant number: 02069-15-RG.

*Correspondence to: Dr. Lin Tao, Department of Oral Biology, College of Dentistry, M/C690, University of Illinois at Chicago, 801 South Paulina Street, Chicago, IL 60612.

E-mail: Ltao@uic.edu 
tercourse. This may decrease both the retention time and load of sexually transmitted pathogens in the vagina. ${ }^{21}$ Second, by comparing douching and HIV prevalence in women, ${ }^{22}$ a study showed that douching with commercial antiseptic preparations is associated with a lower prevalence of HIV, but douching with noncommercial preparations is associated with a higher prevalence of HIV. Therefore, in addition to the flushing effect, the ingredients of douche solutions may also be critical.

Because douching may disrupt the balance of vaginal microbial ecology, its inhibitory effects on vaginal microorganisms, including the commensal lactobacilli, need to be studied. A prior in vitro study using only lactobacilli and antiseptic douche solutions showed that the douches might kill vaginal lactobacilli. ${ }^{23}$ Another in vivo study tested more vaginal microorganisms, but it only analyzed two douche preparations. ${ }^{24}$ Because the vaginal flora contains many different microorganisms in addition to lactobacilli, and because the composition of douche products varies from brand to brand, studies with more douche products and a more complete spectrum of vaginal microorganisms are needed to evaluate the association between douching and vaginal health. In the present study, therefore, we tested in vitro a range of vaginal microorganisms, including vaginal lactobacilli and pathogens associated with $\mathrm{BV}$, vaginal candidiasis (VC), and group B streptococcal vaginitis, in response to seven douche products common to the U.S. market. Among these products, some contained different antiseptics, and some contained none.

\section{MATERIALS AND METHODS}

Seven vaginal douche products were purchased from local department stores or drug stores in the United States. Each product was arbitrarily assigned an alphabetical letter. The ingredients of these products as indicated on their boxes are presented in Table 1. Their $\mathrm{pH}$ values were measured and are also listed in Table 1. The ingredients of douche $\mathrm{B}$ were mixed immediately before use. The ingredients of douches $F$ and $G$ were similar; the difference was that douche $G$ contained additional dyes and fragrance.

The vaginal microorganisms used in this study included a group of normal flora, lactobacilli, and three groups of pathogenic floras: $\mathrm{BV}$-associated
TABLE 1. Ingredients of vaginal douche products tested

\begin{tabular}{|c|c|c|}
\hline Product & Ingredients listed on the package & $\mathrm{pH}^{\mathrm{a}}$ \\
\hline A & Water, vinegar, octoxynol 9 , sorbic acid & 3.0 \\
\hline B & $\begin{array}{l}\text { Medicated douche solution with } \\
\text { Povidone-iodine, final conc. } 0.3 \%\end{array}$ & 3.5 \\
\hline C & $\begin{array}{l}\text { Purified water, sodium chloride, dibasic } \\
\text { sodium phosphate, methyl-paraben, } \\
\text { disodium-EDTA, monobasic sodium } \\
\text { phosphate, sodium lauril sulfate, } \\
\text { propylparaben (post-menstrual) }\end{array}$ & 7.2 \\
\hline $\mathrm{D}$ & Purified water, vinegar, benzoic acid & 3.0 \\
\hline $\mathbf{E}$ & $\begin{array}{l}\text { Purified water, sodium citrate, citric acid, } \\
\text { vinegar }\end{array}$ & 4.0 \\
\hline $\mathrm{F}$ & $\begin{array}{l}\text { Purified water, sodium citrate, citric acid, } \\
\text { vinegar, diazolidinyl urea, octoxynol-9, } \\
\text { cetylpyridinium chloride, edetate } \\
\text { disodium }\end{array}$ & 4.0 \\
\hline G & $\begin{array}{l}\text { Purified water, sodium citrate, citric acid, } \\
\text { SD alcohol } 40 \text {, diazolinidyl urea, } \\
\text { octoxynol-9, fragrance, cetylpyridinium } \\
\text { chloride, edetate disodium, D\&C Red } \\
\text { \#28, FD\&C Blue \#I }\end{array}$ & 4.2 \\
\hline
\end{tabular}

${ }^{\mathrm{a}} \mathrm{pH}$ of the products was not indicated on the packages.

bacteria, group B streptococci, and VC-associated Candida species. To compare the effect of douche products on different vaginal Lactobacillus species, we tested a group of lactobacilli, including eight clinical isolates from a previous study ${ }^{25}$ and three type strains from American Type Culture Collection (ATCC). The group of BV-associated bacteria $^{26}$ included six strains of five genera from ATCC. These included two strains of Gardnerella vaginalis, one strain each of the remaining species, Mobiluncus curtisii subsp. curtisii, Peptostreptococcus tetradius, Mycoplasma hominis, and Ureaplasma urealyticum. Additionally, two strains of group B streptococci (from Dr. Crag Rubens at University of Washington, Seattle) and three species of Candida were also included. A complete list of the microorganisms is shown in Table 2. Multiple species and strains of each group were used for the study in order to compare the range of their sensitivities to various douche products.

The inhibitory effect of the douche products against vaginal microflora were determined by two previously described methods. ${ }^{23}$ The first method measured the minimal inhibitory concentration (MIC) of each douche solution (after proper dilution) required to inactivate individual testing microorganisms. The second method measured the minimal contacting time (MCT) of each douche 
TABLE 2. Minimal inhibitory concentration (MIC, in percentage) of seven commercial douche products against vaginal microorganisms in vitro

\begin{tabular}{|c|c|c|c|c|c|c|c|c|}
\hline \multirow[b]{2}{*}{ Species } & \multirow[b]{2}{*}{ Strains } & \multicolumn{7}{|c|}{ Douche product } \\
\hline & & $\mathrm{A}$ & B & C & $\mathrm{D}$ & $E$ & $\mathrm{~F}$ & G \\
\hline \multicolumn{9}{|c|}{ Lactobacillus clinical isolates } \\
\hline & $\mathrm{KC} 005 \mathrm{~b}$ & 50 & 50 & 50 & 50 & $\mathrm{NE}^{\mathrm{a}}$ & 3.12 & 3.12 \\
\hline & KC 007a & 50 & 50 & 25 & 50 & NE & 3.12 & 3.12 \\
\hline & KC 008 & 50 & NE & so & NE & NE & 3.12 & 3.12 \\
\hline & $\mathrm{KC} 013$ & 50 & 50 & 50 & 50 & NE & 6.25 & 3.12 \\
\hline & $\mathrm{KC} 018 \mathrm{~b}$ & NE & NE & NE & NE & NE & 3.12 & 1.56 \\
\hline & $\mathrm{KC} \mathrm{O2I}$ & 50 & 50 & 25 & 50 & NE & 3.12 & 3.12 \\
\hline & KC 035a & 50 & 50 & 50 & 50 & NE & 6.25 & 6.25 \\
\hline & KC 039 & 50 & 50 & 50 & 50 & 50 & 6.25 & 6.25 \\
\hline \multicolumn{9}{|c|}{ Lactobacillus-type strains } \\
\hline$L$ gasseri & ATCC 9857 & 50 & 50 & 50 & 50 & 50 & 6.25 & 6.25 \\
\hline L. jensenii & ATCC 25258 & NE & NE & 50 & NE & NE & 6.25 & 6.25 \\
\hline L. vaginalis & ATCC 49540 & 50 & 50 & 25 & 50 & 50 & 3.12 & 3.12 \\
\hline \multicolumn{9}{|c|}{ BV-associated bacteria } \\
\hline G. vaginalis & ATCC 14018 & 25 & 25 & 25 & 50 & 50 & 0.39 & 0.39 \\
\hline G. vaginalis & ATCC $49 \mid 45$ & 50 & 50 & 25 & 50 & 50 & 0.78 & 0.78 \\
\hline M. curtisii & ATCC 3524I & 50 & 50 & 25 & 50 & 50 & 0.39 & 0.39 \\
\hline M. hominis & ATCC 23114 & 25 & 25 & 25 & 50 & 50 & 3.12 & 3.12 \\
\hline P. tetradius & ATCC 35098 & 25 & 50 & 25 & 50 & 50 & 1.56 & 1.56 \\
\hline U. urealyticum & ATCC 27168 & 25 & 50 & 12.5 & 50 & 50 & 0.78 & 0.78 \\
\hline \multicolumn{9}{|c|}{ Group B streptococci } \\
\hline S. agalactiae & A909 Type IA & 25 & 50 & 12.5 & 50 & 50 & 0.39 & 0.39 \\
\hline S. agalactiae & $091-2$ & 50 & 50 & 50 & 50 & 50 & 1.56 & 1.56 \\
\hline \multicolumn{9}{|l|}{ Candida species } \\
\hline C. albicans & ATCC 1023I & 50 & 50 & 12.5 & 50 & 50 & 12.5 & 12.5 \\
\hline C. tropicalis & ATCC 13803 & NE & NE & 12.5 & NE & 50 & 12.5 & 12.5 \\
\hline C. glabrato & ATCC 66032 & $\mathrm{NE}$ & NE & 25 & NE & NE & 25 & 25 \\
\hline
\end{tabular}

${ }^{a} \mathrm{NE}$, No inhibitory effect at the maximal concentration (50\%) tested.

solution at solution at full strength without dilution required to inactivate these microorganisms. The inhibitory effect of these douche preparations against vaginal microflora were determined in two different broth media. Lactobacilli were cultured in the Lactobacilli MRS broth (pH 5.5). The BVand $\mathrm{VC}$-associated pathogens and the group $\mathrm{B}$ streptococci were cultured in the Brain Heart Infusion broth (Difco, Detroit, MI)(pH 7.4), supplemented with $5 \%$ horse serum, hemin $(5 \mu \mathrm{g} / \mathrm{ml})$ and vitamin $\mathrm{K} 1(1 \mathrm{ng} / \mathrm{ml})$.

To measure MIC, each bacterial or yeast culture was grown in appropriate medium to midexponential phase. The cultures were centrifuged at $5,000 \mathrm{rpm}$ for $5 \mathrm{~min}$ and washed in phosphatebuffered saline (PBS). Each vaginal douche solution was 2-fold serially diluted (from 50 to $0.09 \%$ ) with appropriate broth medium. The washed bacterial or yeast cells were then inoculated into these different dilutions of broth-douche mixtures at $10^{6}$ colony-forming unit (cfu)/ml. The results for bac- terial growth were observed with a spectrophotometer at $\mathrm{OD}_{600}$ after incubation at $37^{\circ} \mathrm{C}$ for $24 \mathrm{~h}$ under anaerobic condition. The MIC was determined by the lowest concentration of the diluted douche solution that inhibited bacterial growth.

To measure MCT, actively growing bacterial or yeast cultures in mid-exponential phase were centrifuged at 5,000 rpm for $5 \mathrm{~min}$ and washed in phosphate-buffered saline (PBS). The PBS with $\mathrm{pH} 6.5$ was used to wash lactobacilli, whereas the PBS with $\mathrm{pH} 7.3$ was used to wash pathogens. The washed cells were resuspended in an undiluted douche solution at a final concentration of $10^{6} \mathrm{cfu} /$ $\mathrm{ml}$. The cells resuspended in PBS were used as controls. At different time intervals $(0,1,5,10,15$, 20 , and $30 \mathrm{~min}$ ), $0.5-\mathrm{ml}$ aliquots of microorganismdouche suspension were removed, washed in PBS, and resuspended in appropriate broth medium. After anaerobic incubation at $37^{\circ} \mathrm{C}$ for $24 \mathrm{~h}$, the growth of each culture was determined with a spectrophotometer at $\mathrm{OD}_{600}$. 
TABLE 3. Minimum contact times (MCT, in minutes) of seven douche products required to inhibit the growth of vaginal microorganisms in vitro

\begin{tabular}{|c|c|c|c|c|c|c|c|c|}
\hline \multirow[b]{2}{*}{ Species } & \multirow[b]{2}{*}{ Strains } & \multicolumn{7}{|c|}{ Douche product } \\
\hline & & A & B & $\mathrm{C}$ & D & $\mathrm{E}$ & $\mathrm{F}$ & $\mathrm{G}$ \\
\hline \multicolumn{9}{|c|}{ Lactobacillus clinical isolates } \\
\hline & $\mathrm{KC} 005 \mathrm{~b}$ & $N^{a}$ & $<1$ & $<1$ & NE & NE & $<1$ & $<1$ \\
\hline & KC 007a & 10 & $<1$ & $<1$ & NE & NE & $<1$ & $<1$ \\
\hline & KC 008 & 15 & $<1$ & $<1$ & NE & NE & $<1$ & $<1$ \\
\hline & $\mathrm{KC} 013$ & 5 & $<1$ & $<1$ & NE & NE & $<1$ & $<1$ \\
\hline & $\mathrm{KC} 018 \mathrm{~b}$ & NE & $<1$ & $<1$ & NE & NE & $<1$ & $<1$ \\
\hline & KC 02I & 15 & $<1$ & $<1$ & NE & NE & $<1$ & $<1$ \\
\hline & KC 035a & 5 & $<1$ & $<1$ & NE & NE & $<1$ & $<1$ \\
\hline & KC 039 & 15 & $<1$ & $<1$ & NE & NE & $<1$ & $<1$ \\
\hline \multicolumn{9}{|c|}{ Lactobacillus-type strains } \\
\hline L. gasseri & ATCC 9857 & 30 & $<1$ & $<1$ & NE & NE & $<1$ & $<1$ \\
\hline$L$ jensenii & ATCC 25258 & 30 & $<1$ & $<1$ & NE & NE & $<1$ & $<1$ \\
\hline L. vaginalis & ATCC 49540 & NE & $<1$ & $<1$ & NE & NE & $<1$ & $<1$ \\
\hline \multicolumn{9}{|c|}{ BV-associated bacteria } \\
\hline G. vaginalis & ATCC 14018 & $<1$ & $<1$ & $<1$ & $<1$ & inh. $^{b}$ & $<1$ & $<1$ \\
\hline G. vaginalis & ATCC 49145 & $<1$ & $<1$ & $<1$ & $<1$ & $<1$ & $<1$ & $<1$ \\
\hline M. curtisii & ATCC 35241 & $<1$ & $<1$ & $<1$ & 5 & 5 & $<1$ & $<1$ \\
\hline M. hominis & ATCC 23114 & $<1$ & $<1$ & $<1$ & $<1$ & $<1$ & $<1$ & $<1$ \\
\hline P. tetradius & ATCC 35098 & $<1$ & $<1$ & $<1$ & $<1$ & $<1$ & $<1$ & $<1$ \\
\hline U. urealyticum & ATCC 27168 & 30 & $<1$ & $<1$ & NE & NE & $<1$ & $<1$ \\
\hline \multicolumn{9}{|c|}{ Group B streptococci } \\
\hline S. agalactiae & A909 Type IA & 5 & $<1$ & $<1$ & NE & NE & $<1$ & $<1$ \\
\hline S. agalactiae & $091-2$ & 5 & $<1$ & $<1$ & NE & NE & $<1$ & $<1$ \\
\hline \multicolumn{9}{|l|}{ Candida species } \\
\hline C. albicans & ATCC I023I & NE & $<1$ & 5 & inh. & inh. & $<1$ & $<1$ \\
\hline C. tropicalis & ATCC I3803 & NE & $<1$ & $<1$ & inh. & inh. & $<1$ & $<1$ \\
\hline C. glabrata & ATCC 66032 & 30 & $<1$ & $<1$ & inh. & NE & 5 & $<1$ \\
\hline
\end{tabular}

aNE, no inhibitory effect on microbial growth after $30 \mathrm{~min}$ of contact with the douche at full strength.

'Inh., the growth was suppressed, but microorganisms were not killed after 30 min of exposure.

\section{RESULTS}

The $\mathrm{pH}$ values of the seven vaginal douche solutions were determined and listed in Table 1 . The $\mathrm{pH}$ values ranged from 3.0 to 4.2 , except douche $\mathrm{C}$, which was 7.2. The MIC values of the seven douche products against all microorganisms tested are presented in Table 2.

For douches $A$ to $E$, the MIC values against lactobacilli were relatively high, ranging from $25 \%$ to no effect. However, douches $F$ and $G$ exhibited much lower MIC values against the same testing organisms, ranging between $1.56 \%$ and $6.25 \%$. Douches A and B (medicated with povidone-iodine at a final concentration of $0.3 \%$ ) showed no significant effect on the Lactobacillus strains tested. Douches C and D showed no detectable effect against most microorganisms tested. The slight inhibitory effect of douche $\mathrm{C}$ on some vaginal Lactobacillus strains may be due also to its higher $\mathrm{pH}$ (7.2) as compared with the rest of products tested ( $\mathrm{pH}$ range between 3.0 and 4.2). In general, the
MIC values of these douche products against lactobacilli varied from strain to strain.

The MIC values of these douche solutions for $\mathrm{BV}$-associated pathogens were lower than those for lactobacilli. The MIC values for douches $\mathrm{A}$ to $\mathrm{E}$ varied from $12.5 \%$ to $50 \%$. The medicated douche $\mathrm{B}$, designed for use by women with vaginitis symptoms, showed no significant differences from the effects of douches A, C, D, and E. Although douche $\mathrm{C}$ had a higher $\mathrm{pH}$ value, it was more effective against $\mathrm{BV}$-associated pathogens than was the medicated douche B. Douches F and G exhibited significant antibacterial and antifungal activities against all microorganisms tested. The two $G$. vaginalis strains showed different sensitive patterns to these douche products tested. In general, the three yeast cultures were less sensitive to these douche products tested.

The MCT results are presented in Table 3 . The contact time intervals were selected to simulate the actual presence of the douche solution in the va- 
gina. The douche products were tested at full strength without dilution. Douches B, C, F, and G required less than $1 \mathrm{~min}$ to kill all of the tested Lactobacillus strains, BV-associated pathogens, group B streptococci, and 2 of 3 yeast cultures in vitro. No apparent effect on growth of lactobacilli, group B streptococci and $U$. urealyticum was observed after exposure to douches $\mathrm{D}$ and $\mathrm{E}$ for up to $30 \mathrm{~min}$. Interestingly, douches D and E killed most $\mathrm{BV}$-associated pathogens tested. They killed $G$. vaginalis, P. tetradius, and Mycoplasma hominis upon contact for less than $1 \mathrm{~min}$, and Mobiluncus curtisii upon contact for less than $5 \mathrm{~min}$. These two douches also inhibited in vitro the growth of the three Candida species tested. Although douche A suppressed many lactobacilli after 5 to $30 \mathrm{~min}$ of exposure, it more effectively inhibited BVassociated pathogens (less than $1 \mathrm{~min}$ ) and group B streptococci (5 min). But it had virtually no effect on yeasts.

\section{DISCUSSION}

Recent clinical studies suggested that women who frequently douche are associated with an increased risk of pelvic inflammatory disease, ${ }^{7,8} \mathrm{BV},{ }^{9-11}$ chlamydia, ${ }^{12}$ and other sexually transmitted infections, including HIV, ${ }^{13-15}$ miscarriages, ${ }^{16}$ premature birth of low birth-weight infants, ${ }^{17}$ ectopic pregnancy, ${ }^{18-19}$ and cervical cancer. ${ }^{20}$ Nonetheless, data $^{21,22}$ that support douching exist. These include elimination of semen after sexual contact. As a result, it may decrease the retention time and load of sexually transmitted pathogens, presumably reducing the risk of contracting sexually transmitted diseases. Clearly, the effect of douching is controversial, and the mechanism is unknown. Several possibilities might have compromised the research results. First, in some women, douching may be in response to vaginal discomfort caused by preexisting infections. Second, douching may be associated with a high level of sexual activity, which by itself can be a risk factor for vaginal infection. ${ }^{3}$ Third, douching itself may increase the risk of vaginal infection possibly by disturbing vaginal ecology.

Because douching may alter the vaginal ecology by inhibition of the vaginal commensal bacteria, we tested the antimicrobial effects of seven commercial douche products against a list of microorganisms by two assay methods: MIC and MCT. The MCT assay can directly measure the antimicrobial effects of the douche products. ${ }^{23}$ In comparison with MCT, the classical MIC assay might not reflect the actual performance of the douche activity because a douche solution could not remain in the vagina over a 24-hour period, the time used to evaluate inhibition in an MIC assay. But the MIC result can provide information on the antimicrobial potency of various douche products. This information is important because many douche products with different antimicrobial potencies may generate similar MCT results under in vitro assay conditions. Therefore, results from both assays (Tables 2 and 3) may provide complementary information for better evaluation of the potential antimicrobial effect of these douche products.

The results showed that the three products (douches A, D, and E) made of water, vinegar, and other ingredients, had no effect (or mild effect for douche A) on the growth of vaginal lactobacilli, but selectively inhibited multiple vaginal pathogens (Table 3). Four products (douches B, G, F, and G) made of various antiseptics showed a significant inhibitory effect against all vaginal microorganisms tested, including vaginal lactobacilli, BV-associated pathogens, group B streptococci, and three Candida species. Based on these results, therefore, douching might have a varied effect depending upon what product is used and what vaginal conditions exist.

Because most studies ${ }^{7-20}$ have associated douching with various health risks, consumers should be aware that certain douche products may have a negative effect on the vaginal microflora and thus may be harmful to women's health. For women who elect to douche, it may be important to choose an individually appropriate product according to their vaginal health status. We speculate that if a woman with a healthy, Lactobacillus-dominant vagina douches with a solution containing antiseptics, her lactobacilli can be eliminated and other bacteria may colonize and overgrow. On the other hand, if a woman does not have vaginal lactobacilli, or if she already has an infection, a douche product containing antiseptics may conceivably help decrease the vaginal load of infecting bacteria. We also speculate that after the antimicrobial treatment, it may be helpful to apply a second product to facilitate the reestablishment of vaginal lactobacilliperhaps a Lactobacillus vaginal suppository or acid gel. 
In summary, this in vitro study suggested that the antimicrobial effects of the commercial douche products varied among different brands and microbial species tested. The antiseptic-containing douche products inhibited all microorganisms tested. The vinegar-containing products selectively inhibited pathogens associated with vaginal infections, but not vaginal lactobacilli.

\section{ACKNOWLEDGMENT}

We would like to thank Dr. Grag Rubens of University of Washington, Seattle for the gift of group B streptococci.

\section{REFERENCES}

1. Larsen B. Vaginal flora in health and disease. Clin Obstet Gynecol 1993;36:107-121.

2. Klebanoff SJ, Hillier SL, Eschenbach DA, Waltersdorph AM. Control of the microbial flora of the vagina by $\mathrm{H}_{2} \mathrm{O}_{2}$ generating lactobacilli. J Infect Dis 1991; 164:94-100.

3. Rosenberg MJ, Phillips RS, Holms MD. Vaginal douching. Who and why? J Reprod Med 1991;36:753-758.

4. Aral SO, Mosher WD, Cates W Jr. Vaginal douching among women of reproductive age in the United States: 1988. Am J Public Health 1992;82:210-214.

5. Chacko MR, McGill L, Johnson TC, Smith PB, Nenney SW. Vaginal douching in teenagers attending a family planning clinic. J Adolesc Health Care 1989;10:217-219.

6. Merchant JS, Oh K, Klerman LV. Douching: a problem for adolescent girls and young women. Arch Pediatr Adolesc Med 1999;153:834-837.

7. Scholes D, Daling JR, Stergachis A, Weiss NS, Wang SP, Grayston JT. Vaginal douching as a risk factor for acute pelvic inflammatory disease. Obstet Gynecol 1993;81:601-606.

8. Wolner-Hanssen P, Eschenbach DA, Paavonen J, et al. Association between vaginal douching and acute pelvic inflammatory disease. JAMA. 1990;263:1936-1941.

9. Hawes SE, Hillier SL, Benedetti J, et al. Hydrogen peroxide-producing lactobacilli and acquisition of vaginal infections. J Infect Dis 1996;174:1058-1063.

10. Harwood B, Mittedorf R, Judge D, Dayal S, Walker C. Patterns of vaginal douching and their association with vaginal bacteriosis. Infect Dis Ob Gyn. 1996;4:51.

11. Rajamanoharan S, Low N, Jones SB, Pozniak AL. Bacterial vaginosis, ethnicity, and the use of genital cleaning agents: a case control study. Sex Transm Dis 1999; 26:404-409.

12. Scholes D, Stergachis A, Ichikawa LE, Heidrich FE,
Holmes KK, Stamm WE. Vaginal douching as a risk factor for cervical Chlamydia trachomatis infection. Obstet Gynecol 1998;91:993-997.

13. La Ruche G, Messou N, Ali-Napo L, et al. Vaginal douching: association with lower genital tract infections in African pregnant women. Sex Transm Dis 1999;26: 191-196.

14. Joesoef MR, Sumampouw H, Linnan M, Schmid S, Idajadi A, St Louis ME. Douching and sexually transmitted diseases in pregnant women in Surabaya, Indonesia. Am J Obstet Gynecol 1996;174:115-119.

15. Foxman B, Aral SO, Holmes KK. Interrelationships among douching practices, risky sexual practices, and history of self-reported sexually transmitted diseases in an urban population. Sex Transm Dis 1998;25:90-99.

16. Llahi-Camp JM, Rai R, Ison C, Regan L, TaylorRobinson $\mathrm{D}$. Association of bacterial vaginosis with a history of second trimester miscarriage. Hum Reprod 1996;11:1575-1578.

17. Fiscella K, Franks P, Kendrick JS, Bruce FG. The risk of low birth weight associated with vaginal douching. Obstet Gynecol 1998;92:913-917.

18. Kendrick JS, Atrash HK, Strauss LT, Gargiullo PM, Ahn YW. Vaginal douching and the risk of ectopic pregnancy among black women. Am J Obstet Gynecol 1997; 176:991-997.

19. Chow WH, Daling JR, Weiss NS, Moore DE, Soderstrom R. Vaginal douching as a potential risk factor for tubal ectopic pregnancy. Am J Obstet Gynecol 1985; 153:727-729.

20. Gardner JW, Schuman KI, Slattery ML, Sanborn JS, Abbott 'TM, Overall JC Jr. Is vaginal douching related to cervical carcinoma? Am J Epidemiol 1991;133:368-375.

21. Tevi-Benissan $\mathrm{C}$, Belec L, Levy $M$, et al. In vivo semen-associated $\mathrm{pH}$ neutralization of cervicovaginal secretions. Clin Diagn Lab Immunol 1997;4:367-374.

22. Gresenguet G, Kreiss JK, Chapko MK, Hillier SL, Weiss NS. HIV infection and vaginal douching in central Africa. AIDS 1997;11:101-106.

23. Juliano C, Piu L, Gavini E, Zanetti S, Fadda G. In vitro antibacterial activity of antiseptics against vaginal lactobacilli. Eur J Clin Microbiol Infect Dis 1992;11:11661169.

24. Onderdonk AB, Delaney ML, Hinkson PL, DuBois AM. Quantitative and qualitative effects of douche preparations on vaginal microflora. Obstet Gynecol 1992;80:333-338.

25. Pavlova SI, Kilic AO, Mou SM, Tao L. Phage infection in vaginal lactobacilli: an in vitro study. Infect $\mathrm{Dis} \mathrm{Ob}$ stet Gynecol 1997;5:36-44.

26. Spiegel CA. Bacterial vaginosis. Clin Microbiol Rev 1991;4:485-502. 


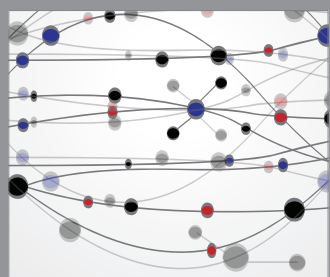

The Scientific World Journal
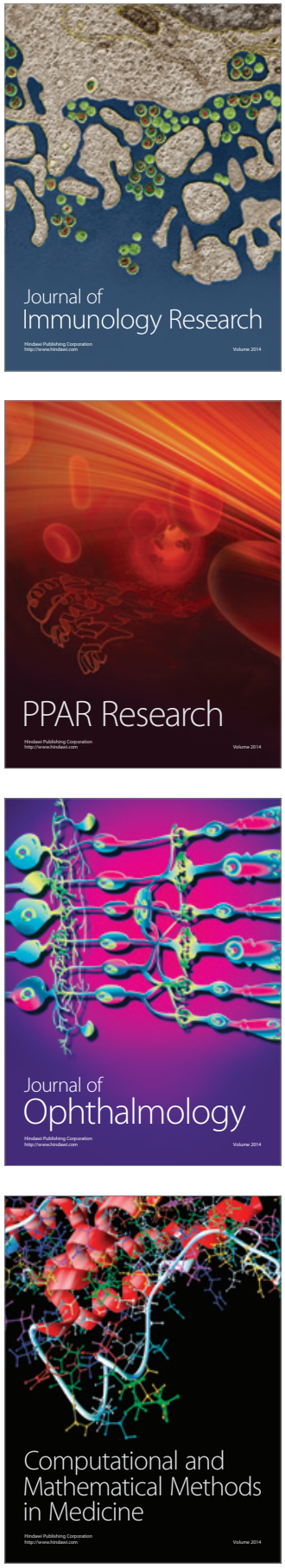

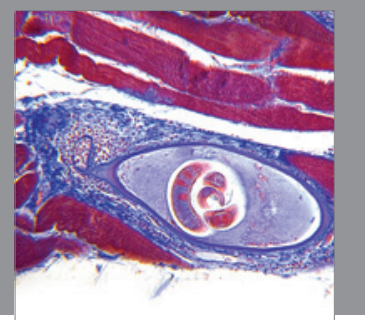

Gastroenterology

Research and Practice
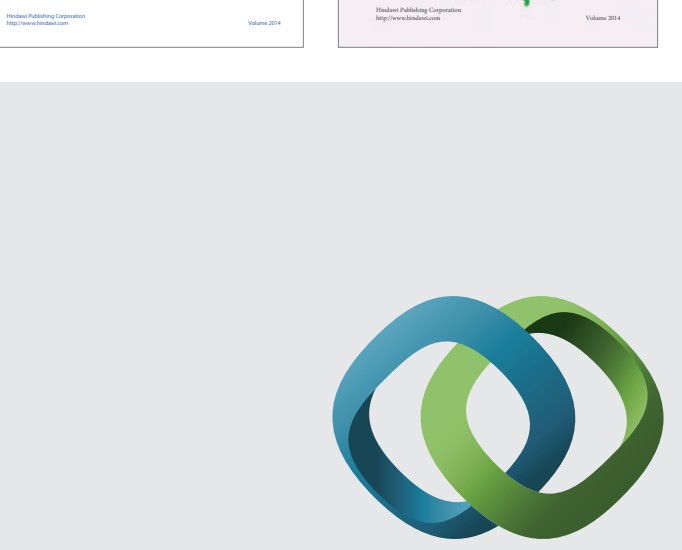

\section{Hindawi}

Submit your manuscripts at

http://www.hindawi.com
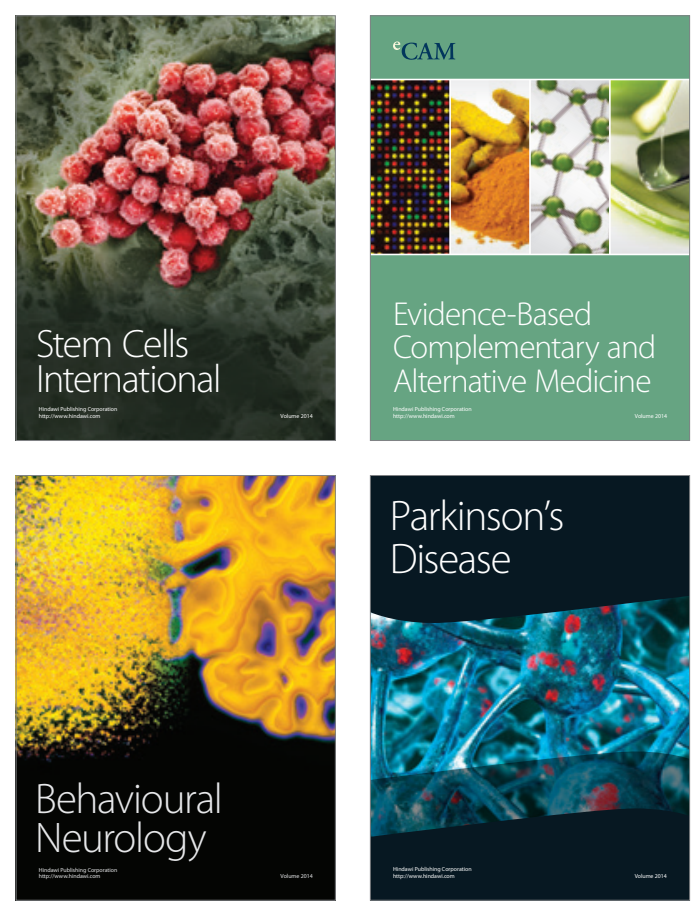

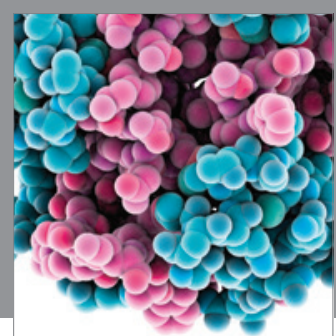

Journal of
Diabetes Research

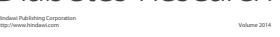

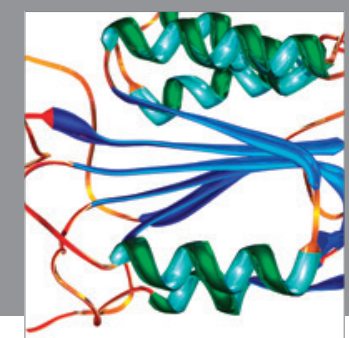

Disease Markers
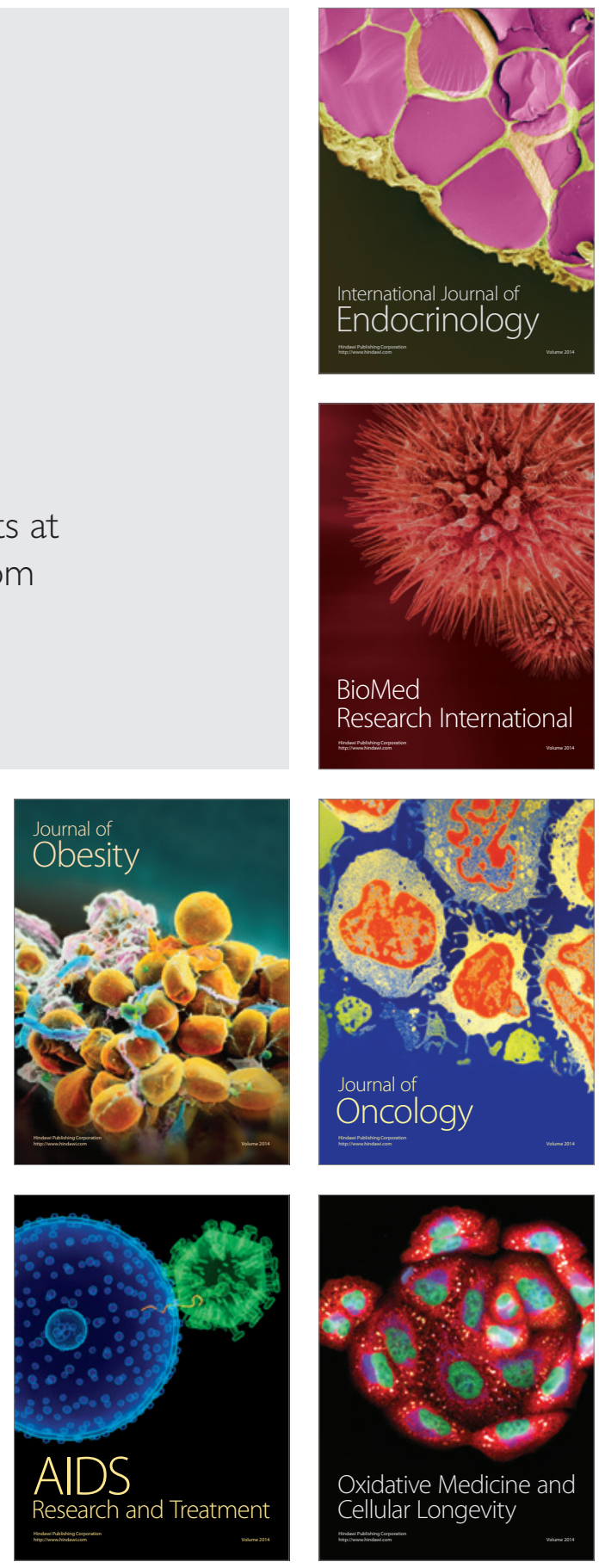